\title{
Strict dissipativity implies turnpike behavior for time-varying discrete time optimal control problems
}

Lars Grüne, Simon Pirkelmann, and Marleen Stieler

\begin{abstract}
We consider the turnpike property for infinite horizon undiscounted optimal control problems in discrete time and with time-varying data. We show that, under suitable conditions, a time varying strict dissipativity notion implies the turnpike property and a continuity property of the optimal value function. We also discuss the relation of strict dissipativity to necessary optimality conditions and illustrate our results by an example.
\end{abstract}

\section{Introduction}

Infinite horizon optimal control problems are notoriously difficult to solve if the problem data is time-varying. Unlike the time invariant case, global approaches like dynamic programming do not lead to a stationary Bellman equation but - in the discrete time setting considered in this paper — rather to an infinite sequence of such equations. Since we consider undiscounted problems in this paper, the dynamic programming approach has the additional difficulty that the Bellman equation is not a contraction. Pontryagin-type necessary optimality conditions (see, e.g., [2, 4]) appear somewhat more suitable for this class of problems, however, they still lead to an infinite dimensional system of coupled difference equations for which no general solution method exists.

It has been observed in various papers (e.g., in $[1,18,19])$, that the turnpike property facilitates the computation of optimal trajectories on long finite time horizons. In the time-invariant setting of these papers, the turnpike property, which has its origins in mathematical economy $[6,15]$, describes the fact that an optimal trajectory on a finite time horizon stays close to an optimal equilibrium most of the time. In or-

Lars Grüne (lars.gruene@uni-bayreuth.de), Simon Pirkelmann (simon.pirkelmann@unibayreuth.de) and Marleen Stieler (marleen.stieler@uni-bayreuth.de) are with the Chair of Applied Mathematics, Mathematical Institute, University of Bayreuth, 95440 Bayreuth, Germany. The research was supported by the DFG Grants GR1569/13-1 and 16-1. 
der to compute an (approximately) optimal trajectory, it thus suffices to compute the optimal equilibrium as well as optimal paths to and from the optimal equilibrium. For the infinite horizon problem, the turnpike property demands that the optimal trajectory converges to the optimal equilibrium. Under suitable conditions, the finite horizon turnpike property holds if and only if the infinite horizon turnpike property holds [10].

In the time-varying setting of this paper, the optimal equilibrium is replaced by a time-varying infinitely long trajectory, at which the system is operated optimally in an overtaking sense. Since this trajectory is very difficult to compute, compared to the time-invariant setting the situation reverses: instead of using the turnpike property and the knowledge about the optimal equilibrium for the approximation of finite horizon optimal trajectories, now we may use finite horizon optimal trajectories (which can be efficiently computed numerically if the horizon is not too long) and the turnpike property in order to approximate the infinite-horizon optimal trajectory. This can be done directly by numerically computing optimal trajectories on finite horizons with increasing length, or indirectly via a receding horizon or model predictive control (MPC) approach, see Remark 1 and [12]. However, in order to decide whether these methods can be employed, we need to find ways to check whether the given optimal control problem exhibits the turnpike property.

In the time-invariant case it is known that there is a strong relation between strict dissipativity in the sense of Willems [20] and the turnpike property, see [11]. The main result in this paper shows that under suitable conditions a time-varying version of strict dissipativity implies the time-varying turnpike property. Moreover, we show that together with a local controllability assumption this property also implies a continuity property for the optimal value function which is useful for the analysis of MPC schemes. We finally discuss the relation between strict dissipativity and necessary optimality conditions for uniformly convex problems and illustrate our results by a simple yet nontrivial example.

\section{Problem statement and definitions}

\subsection{Setting}

Consider the following time-varying control system

$$
x(k+1)=f(k, x(k), u(k)), \quad x\left(k_{0}\right)=x_{0},
$$

with $f: \mathbb{N}_{0} \times X \times U \rightarrow X$ and normed spaces $X$ and $U$. In this setting $k \in \mathbb{N}_{0}$ represents a time instant, $x(k) \in X$ is the state of the system at that time and $u(k) \in U$ is the control applied to the system during the next sampling interval. For a given initial state $x_{0} \in X$ at initial time $k_{0}$ and a control sequence $u \in U^{N}$ of length $N \in \mathbb{N}$ we denote the state trajectory which results from iteratively applying $(1)$ by $x_{u}\left(\cdot ; k_{0}, x_{0}\right)$. 
To shorten the notation we may omit the initial time when it is clear from the context and write $x_{u}\left(\cdot, x_{0}\right)$ instead.

We define $\mathbb{X}(k) \subseteq X$ to be the sets of admissible states at time $k$ and $\mathbb{U}(k, x) \subseteq U$ as the set of admissible control values for $x \in \mathbb{X}(k)$.

We denote by $\mathbb{U}^{N}(k, x)$ the set of admissible control sequences for initial state $x \in \mathbb{X}(k)$, i.e. control sequences $u \in U^{N}$ that satisfy

$$
u(j) \in \mathbb{U}\left(k+j, x_{u}(j ; k, x)\right) \text { and } x_{u}(j+1 ; k, x) \in \mathbb{X}(k+j+1)
$$

for all $j \in\{0, \ldots, N-1\}$ and similarly $\mathbb{U}^{\infty}(k, x)$ as the set of control sequences $u \in U^{\infty}$ satisfying

$$
u(j) \in \mathbb{U}\left(k+j, x_{u}(j ; k, x)\right) \text { and } x_{u}(j+1 ; k, x) \in \mathbb{X}(k+j+1)
$$

for all $j \in \mathbb{N}_{0}$.

The goal in our setting is to investigate the structure and properties of solutions to the infinite-horizon optimal control problem

$$
\underset{u \in \mathbb{U}^{\infty}\left(k_{0}, x_{0}\right)}{\operatorname{minimize}} \underbrace{\sum_{j=0}^{\infty} \ell\left(k_{0}+j, x_{u}\left(j ; k_{0}, x_{0}\right), u(j)\right)}_{=: J_{\infty}\left(k_{0}, x_{0}, u\right)},
$$

where $\ell: \mathbb{N}_{0} \times X \times U \rightarrow \mathbb{R}$ is the stage cost function.

\subsection{Overtaking optimality}

The objective function in (2) will not necessarily assume a finite value for all control sequences $u \in U^{\infty}$. In particular it may happen that $J_{\infty}(k, x, u)=-\infty$ for several control sequences $u \in U^{\infty}$, i.e. we do not get a unique minimal value which means it is not obvious how to decide which control sequence performs best. Similarly, it may happen that $J_{\infty}(k, x, u)=\infty$ for all control sequences in which case the usual definition of optimality also breaks down. To deal with this issue we use the concept of overtaking optimality ${ }^{1}$ which was first introduced by Gale in [7].

Definition 1 (Overtaking optimality) Let $x \in \mathbb{X}(k)$ and consider a control sequence $u^{*} \in \mathbb{U}^{\infty}(k, x)$ with corresponding state trajectory $x_{u^{*}}(\cdot ; k, x)$. The pair $\left(x_{u^{*}}, u^{*}\right)$ is called overtaking optimal if

$$
\liminf _{K \rightarrow \infty} \sum_{j=0}^{K-1} \ell\left(k+j, x_{u}(j ; k, x), u(j)\right)-\ell\left(k+j, x_{u^{*}}(j), u^{*}(j)\right) \geq 0
$$

\footnotetext{
${ }^{1}$ In particular in the economic literature, this property is also referred to as catching up optimality, see e.g. [3].
} 
for all $u \in \mathbb{U}^{\infty}(k, x)$.

Using the above definition we can handle the case of infinite values of $J_{\infty}(k, x, u)$. The definition considers a trajectory pair $\left(x_{u^{*}}, u^{*}\right)$ as optimal if in the limit inferior its cost is overtaken by the cost of any other trajectory. If the optimal value is finite, overtaking optimality coincides with the 'usual' definition of optimality. The following definition characterizes for which trajectory the system yields optimal performance, where optimality is now thought of in the sense of Definition 1. Note that both definitions just differ in the fact, that in the second one the initial value is no longer fixed.

Definition 2 (Optimal operation) Let $x \in \mathbb{X}(k)$ and consider a control sequence $u^{*} \in \mathbb{U}^{\infty}(k, x)$ with corresponding state trajectory $x^{*}=x_{u^{*}}(\cdot ; k, x)$. We say the system (1) is optimally operated at $\left(x^{*}, u^{*}\right)$ if

$$
\liminf _{K \rightarrow \infty} \sum_{j=0}^{K-1} \ell\left(k+j, x_{u}\left(j ; k, x^{\prime}\right), u(j)\right)-\ell\left(k+j, x^{*}(j), u^{*}(j)\right) \geq 0
$$

for all $x^{\prime} \in \mathbb{X}(k)$ and $u \in \mathbb{U}^{\infty}\left(k, x^{\prime}\right)$.

To better understand the difference between both definitions it is insightful to consider the second definition from a viewpoint of a time-invariant setting where there exists an optimal equilibrium at which the system performs best. In our setting the optimal equilibrium corresponds to a more general time-varying pair $\left(x^{*}, u^{*}\right)$ that is defined in Definition 2, whereas the first definition formally introduces the optimality notion we are using.

In the subsequent sections we will always assume that a trajectory pair $\left(x^{*}, u^{*}\right)$ at which the system is optimally operated exists.

\section{Definitions of turnpike and continuity property}

We will consider two different versions of the turnpike property, one for the finite and one for the infinite-horizon optimal control problem. In order to be able to treat both in a unified way without having to distinguish between the optimality notions on finite or infinite horizon we introduce a shifted cost functional, which always has a finite value along the optimal trajectory.

Definition 3 (Shifted stage cost) We define the shifted stage cost $\hat{\ell}: \mathbb{N}_{0} \times X \times U \rightarrow$ $\mathbb{R}$ as

$$
\hat{\ell}(k, x, u):=\ell(k, x, u)-\ell\left(k, x^{*}(k), u^{*}(k)\right)
$$

and the shifted cost functional as

$$
\hat{J}_{N}(k, x, u):=\sum_{j=0}^{N-1} \hat{\ell}\left(k+j, x_{u}(j ; k, x), u(j)\right)
$$


for $N \in \mathbb{N} \cup\{\infty\}$. The corresponding optimal value function is given by

$$
\hat{V}_{N}(k, x):=\inf _{u \in \mathbb{U}^{N}(k, x)} \hat{J}_{N}(k, x, u)=\inf _{u \in \mathbb{U}^{N}(k, x)} J_{N}(k, x, u)-J_{N}^{*}(k)=V_{N}(k, x)-J_{N}^{*}(k),
$$

with $J_{N}^{*}(k):=\sum_{j=k}^{k+N-1} \ell\left(j, x^{*}(j), u^{*}(j)\right)$.

With this definition we are now able to define the turnpike property on finite and infinite time horizons. In the following we will write

$$
|(x, u)|_{(\bar{x}, \bar{u})}:=\|x-\bar{x}\|+\|u-\bar{u}\|
$$

to shorten the notation, using the norms on the spaces $X$ and $U$.

Definition 4 (Time-varying turnpike property) Consider a trajectory pair $\left(x^{*}, u^{*}\right)$ at which the system (1) is optimally operated.

a) The optimal control problem on infinite horizon with shifted stage cost $\hat{\ell}$ has the time-varying turnpike property at $\left(x^{*}, u^{*}\right)$ if the following holds: There exists $\rho \in \mathscr{L}^{2}$ such that for each $k \in \mathbb{N}_{0}$, each optimal trajectory $x_{u_{\infty}^{*}}(\cdot, x), x \in \mathbb{X}(k)$ and all $P \in \mathbb{N}$ there is a set $\mathscr{Q}(k, x, P, \infty) \subseteq \mathbb{N}_{0}$ with $\# \mathscr{Q}(k, x, P, \infty) \leq P$ and

$$
\left|\left(x_{u_{\infty}^{*}}(j ; k, x), u_{\infty}^{*}(j)\right)\right|_{\left(x^{*}(k+j), u^{*}(k+j)\right)} \leq \rho(P)
$$

for all $j \in \mathbb{N}_{0}$ with $j \notin \mathscr{Q}(k, x, P, \infty)$.

b) The optimal control problem on finite horizon has the time-varying turnpike property at $\left(x^{*}, u^{*}\right)$ if the following holds: There exists $\sigma \in \mathscr{L}$ such that for each $k \in \mathbb{N}_{0}$, each optimal trajectory $x_{u_{N}^{*}}(\cdot, x), x \in \mathbb{X}(k)$ and all $N, P \in \mathbb{N}$ there is a set $\mathscr{Q}(k, x, P, N) \subseteq\{0, \ldots, N\}$ with $\# \mathscr{Q}(k, x, P, N) \leq P$ and

$$
\left|\left(x_{u_{N}^{*}}(j ; k, x), u_{N}^{*}(j)\right)\right|_{\left(x^{*}(k+j), u^{*}(k+j)\right)} \leq \sigma(P)
$$

for all $j \in\{0, \ldots, N\}$ with $j \notin \mathscr{Q}(k, x, P, N)$.

The turnpike property describes the fact that optimal solutions on the infinite and finite horizon are close to the optimal trajectory of the system most of the time. This is illustrated in Figure 1 for the finite-horizon case.

Definition 5 (Continuity property of $\hat{V}_{N}$ and $\hat{V}_{\infty}$ ) The optimal value functions $\hat{V}_{N}$ and $\hat{V}_{\infty}$ are (approximately) continuous at $x^{*}$ if for each $k \in \mathbb{N}_{0}$ there is an open ball $\mathscr{B}_{\varepsilon}\left(x^{*}(k)\right), \varepsilon>0$, around $x^{*}(k)$ and a function $\gamma_{V}: \mathbb{R}_{0}^{+} \times \mathbb{R}_{0}^{+} \rightarrow \mathbb{R}_{0}^{+}$with $\gamma_{V}(N, r) \rightarrow$ 0 if $N \rightarrow \infty$ and $r \rightarrow 0$, and $\gamma_{V}(\cdot, r), \gamma_{V}(N, \cdot)$ monotonous for fixed $r$ and $N$, such that for all $x \in \mathscr{B}_{\varepsilon}\left(x^{*}(k)\right) \cap \mathbb{X}(k)$ and all $N \in \mathbb{N} \cup\{\infty\}$ the inequality

$$
\left|\hat{V}_{N}(k, x)-\hat{V}_{N}\left(k, x^{*}(k)\right)\right| \leq \gamma_{V}\left(N,\left\|x-x^{*}(k)\right\|\right)
$$

$2 \mathscr{L}:=\left\{\sigma: \mathbb{R}_{0}^{+} \rightarrow \mathbb{R}_{0}^{+} \mid \sigma\right.$ is continuous and strictly decreasing with $\left.\lim _{s \rightarrow \infty} \sigma(s)=0\right\}$, cf. [14]. 


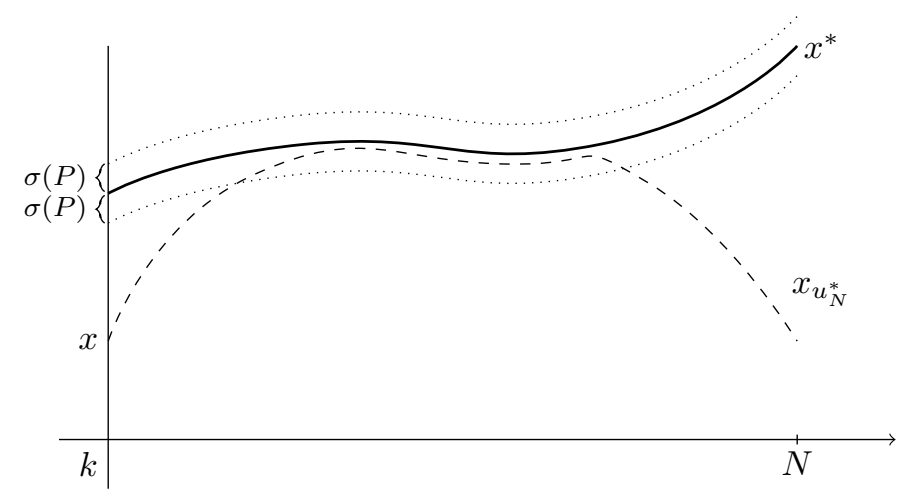

Fig. 1 Finite horizon turnpike property for time-varying systems.

holds, where we make the assumption that $\gamma_{V}(\infty, r)=: \omega_{V}(r)$ with $\omega_{V} \in \mathscr{K}_{\infty}{ }^{3}$.

Remark 1. As mentioned in the introduction, the turnpike property is not only an interesting phenomenon in general system theory and allows to relate finite and infinite horizon optimal trajectories, but also plays an important role in the context of model predictive control (MPC). In this control method, a control input is synthesized by iteratively solving finite horizon optimal control problems and concatenating the initial pieces of the resulting optimal trajectories. In particular, the turnpike property guarantees that the optimal open-loop trajectories, which are calculated in the MPC iterations, are close to the infinite horizon optimal trajectory for a certain number of time steps. Together with continuity of the optimal value function, this allows for the construction of a Lyapunov function as well as convergence and performance estimates for time-invariant MPC, see [8] and [13], and for performance estimates of the MPC closed-loop solution in the time-varying setting, see [12].

\section{From dissipativity to turnpike}

While the turnpike and continuity properties are handy tools to use in the construction of approximately optimal trajectories and for the analysis of MPC schemes, they are in general difficult to verify directly. As an alternative we consider the concept of dissipativity ${ }^{4}$, which is a property of the system that can be checked more easily. Our goal in this section is to prove that the turnpike and continuity properties are satisfied if we assume that the system is (strictly) dissipative as follows.

${ }^{3} \mathscr{K}_{\infty}:=\left\{\alpha: \mathbb{R}_{0}^{+} \rightarrow \mathbb{R}_{0}^{+} \mid \alpha\right.$ is continuous, strictly increasing and unbounded with $\left.\alpha(0)=0\right\}$

${ }^{4}$ Introduced in the context of control systems by Jan Willems in 1972, see [20]. 
Definition 6 (Dissipativity) The system (1) is dissipative with respect to the supply rate $s: \mathbb{N}_{0} \times X \times U$ if there exists a storage function $\lambda: \mathbb{N}_{0} \times X \rightarrow \mathbb{R}$ bounded from below on $X$ such that for all $k \in \mathbb{N}_{0}$ and all $(x, u) \in \mathbb{X}(k) \times \mathbb{U}(k, x)$ the following holds:

$$
\lambda(k+1, f(k, x, u))-\lambda(k, x) \leq s(k, x, u) .
$$

The system (1) is strictly dissipative with respect to the supply rate $s: \mathbb{N}_{0} \times X \times U$ and the optimal trajectory $\left(x^{*}, u^{*}\right)$, if there exists $\alpha \in \mathscr{K}_{\infty}$ such that

$$
\lambda(k+1, f(k, x, u))-\lambda(k, x) \leq s(k, x, u)-\alpha\left(|(x, u)|_{\left(x^{*}(k), u^{*}(k)\right)}\right)
$$

holds for all $k \in \mathbb{N}_{0}$ and all $(x, u) \in \mathbb{X}(k) \times \mathbb{U}(k, x)$.

In the sequel we will assume that the system (1) is strictly dissipative with respect to the supply rate $s(k, x, u)=\hat{\ell}(k, x, u)=\ell(k, x, u)-\ell\left(k, x^{*}(k), u^{*}(k)\right)$. We further assume that the optimal trajectory $x^{*}$ from Definition 2 is cheaply reachable, which expresses that it can be reached from any initial state with bounded cost. Since the shifted cost along $x^{*}$ is 0 , this can be expressed via a bound on the shifted optimal value functions.

Assumption 1 (Cheap reachability) The trajectory pair $\left(x^{*}, u^{*}\right)$ is called cheaply reachable if there exists $E \in \mathbb{R}$ such that for each $k \in \mathbb{N}_{0}$ and for all $x \in \mathbb{X}(k)$, $N \in \mathbb{N} \cup\{\infty\}$ the inequality

$$
\hat{V}_{N}(k, x) \leq E
$$

holds.

Using dissipativity and cheap reachability it can be shown that both the finite and infinite optimal control problems have the turnpike property from Section 3.

Theorem 1 (Strict dissipativity and cheap reachability imply turnpike)

Let $\left(x^{*}, u^{*}\right)$ be an optimal pair. If the optimal control problem is strictly dissipative wrt the supply rate $s(k, x, u)=\hat{\ell}(k, x, u)=\ell(k, x, u)-\ell\left(k, x^{*}(k), u^{*}(k)\right)$ with bounded storage function $\lambda$ for the trajectory pair $\left(x^{*}, u^{*}\right)$ and $\left(x^{*}, u^{*}\right)$ is cheaply reachable then the turnpike property from Definition 4 holds.

Proof. We first prove the finite-horizon turnpike property from Definition 4 (b). Let $k \in \mathbb{N}_{0}, x \in \mathbb{X}(k)$ and consider a control sequence $u \in \mathbb{U}(k, x)$ with corresponding state trajectory $x_{u}(\cdot ; k, x)$. From strict dissipativity we have

$$
\begin{aligned}
\hat{\ell}\left(k+j, x_{u}(j ; k, x), u(j)\right) \geq & \lambda\left(k+j+1, f\left(k+j, x_{u}(j ; k, x), u(j)\right)\right)-\lambda\left(k+j, x_{u}(j)\right) \\
& +\alpha\left(\left|\left(x_{u}(j ; k, x), u(j)\right)\right|_{\left(x^{*}(j), u^{*}(j)\right)}\right)
\end{aligned}
$$

for all $j \in \mathbb{N}_{0}$. Summing for $j=0, \ldots, N-1$ yields 


$$
\begin{aligned}
\hat{J}_{N}(k, x, u) & =\sum_{j=0}^{N-1} \hat{\ell}\left(k+j, x_{u}(j ; k, x), u(j)\right) \\
& \geq \lambda\left(k+N, f\left(k+N-1, x_{u}(N-1 ; k, x), u(N-1)\right)\right)-\lambda\left(k, x_{u}(k ; k, x)\right) \\
& +\sum_{j=0}^{N-1} \alpha\left(\left|\left(x_{u}(j ; k, x), u(j)\right)\right|_{\left(x^{*}(j), u^{*}(j)\right)}\right) .
\end{aligned}
$$

We prove the finite-horizon turnpike property by contradiction. Suppose the turnpike property does not hold for

$$
\sigma(P):=\alpha^{-1}\left(\frac{2 M_{\lambda}+E}{P}\right)
$$

where $M_{\lambda}>0$ is a bound on $|\lambda|$ and with $E$ from Assumption 1. This means that there is $N \in \mathbb{N}, x \in \mathbb{X}(k)$ and $P \in \mathbb{N}$ such that the number of elements $j \in$ $\mathscr{Q}(k, x, P, N)$, i.e. those elements for which $\left|\left(x_{u_{N}^{*}}(j ; k, x), u_{N}^{*}(j)\right)\right|_{\left(x^{*}(j), u^{*}(j)\right)}>\sigma(P)$ is larger than $P$. Using (8) with the optimal control sequence $u=u_{N}^{*}$ and taking only those elements in the sum into account for which $\left|\left(x_{u_{N}^{*}}(j ; k, x), u_{N}^{*}(j)\right)\right|_{\left(x^{*}(j), u^{*}(j)\right)}>$ $\sigma(P)$ holds (the other summands are lower-bounded by zero), this implies

$$
\hat{V}_{N}(k, x)=\hat{J}_{N}\left(k, x, u_{N}^{*}\right)>-2 M_{\lambda}+P \alpha(\sigma(P))=-2 M_{\lambda}+2 M_{\lambda}+E=E .
$$

However, this contradicts Assumption 1.

The proof for the infinite horizon follows analogously with

$$
\rho(P):=\alpha^{-1}\left(\frac{2 M_{\lambda}+E}{P}\right) .
$$

To show that not only the turnpike property but also continuity of the optimal value function holds we need some additional assumptions, first of all local controllability near the optimal trajectory of the system.

Assumption 2 (Local controllability) Assume that the system is locally controllable along the trajectory pair $\left(x^{*}, u^{*}\right)$, i.e. there exists a time $d \in \mathbb{N}, \delta_{c}>0$, and $\gamma_{x}, \gamma_{u}, \gamma_{c} \in \mathscr{K}_{\infty}$ such that for each $k \in \mathbb{N}_{0}$ and for any two points $x \in \mathscr{B}_{\delta_{c}}\left(x^{*}(k)\right)$, $y \in \mathscr{B}_{\delta_{c}}\left(x^{*}(k+d)\right)$ there exists $u \in \mathbb{U}^{d}(x)$ satisfying $x_{u}(d, x)=y$ and the estimates $\left\|x_{u}(j ; k, x)-x^{*}(k+j)\right\| \leq \gamma_{x}(\delta),\left\|u(j)-u^{*}(k+j)\right\| \leq \gamma_{u}(\delta)$ and $\mid \hat{\ell}(j+$ $\left.k, x_{u}(j ; k, x), u(j)\right) \mid \leq \gamma_{c}(\delta)$ for all $j=0, \ldots, d-1$, where $\delta:=\max \left\{\left\|x-x^{*}(k)\right\|, \| y-\right.$ $\left.x^{*}(k+d) \|\right\}$.

Clearly, local controllability means that any two points within a tube along the optimal trajectory can be connected in forward time as illustrated by Figure 2. The following definition is closely related to strict dissipativity. The cost function $\tilde{\ell}$ defined therein is sometimes also called rotated stage cost. 


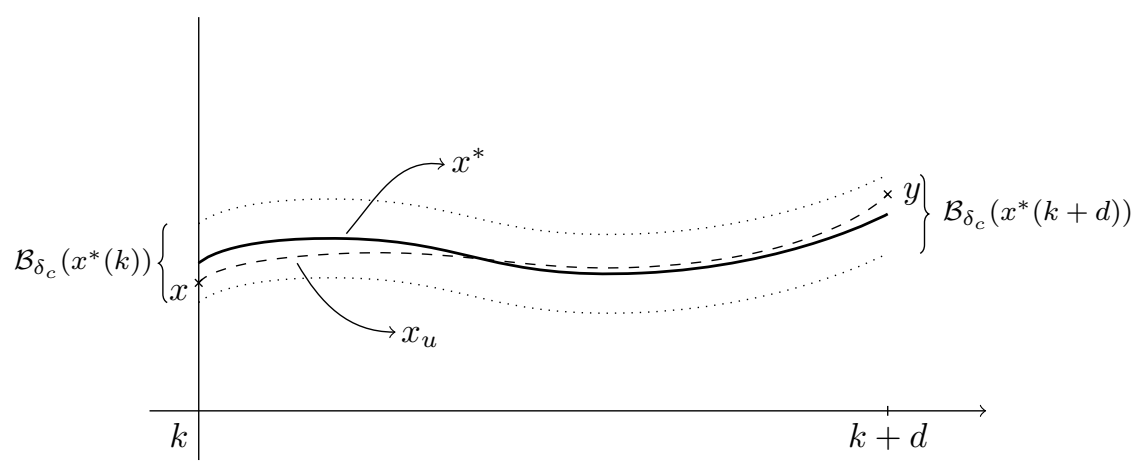

Fig. 2 Local controllability along the optimal trajectory.

Definition 7 (Modified stage cost) Consider the modified stage cost $\tilde{\ell}: \mathbb{N}_{0} \times X \times$ $U \rightarrow \mathbb{R}_{\geq 0}$ defined by:

$$
\tilde{\ell}(k, x, u):=\hat{\ell}(k, x, u)+\lambda(k, x)-\lambda(k+1, f(k, x, u))
$$

using the storage function $\lambda$ from the assumed strict dissipativity of the system. We also define the modified cost functional by

$$
\tilde{J}_{N}(k, x, u):=\sum_{j=0}^{N-1} \tilde{\ell}\left(k+j, x_{u}(j ; k, x), u(j)\right)
$$

Note that the modified stage cost is bounded from below by a function $\alpha_{l} \in \mathscr{K}_{\infty}$, i.e.

$$
\tilde{\ell}(k, x, u) \geq \alpha_{l}\left(|(x, u)|_{\left(x^{*}(k), u^{*}(k)\right)}\right)
$$

holds for all $(x, u) \in \mathbb{X}(k) \times \mathbb{U}(k, x)$. This is immediately concluded from strict dissipativity of the system, with $\alpha_{l}:=\alpha$. One easily sees that for the modified cost functional the following identity holds:

$$
\tilde{J}_{N}(k, x, u)=\hat{J}_{N}(k, x, u)+\lambda(k, x)-\lambda\left(k+N, x_{u}(N ; k, x)\right) .
$$

Assumption 3 There exists an upper bound $\alpha_{u} \in \mathscr{K}_{\infty}$ such that the modified stage cost from Defintion 7 satisfies the inequality

$$
\tilde{\ell}(k, x, u) \leq \alpha_{u}\left(\|(x, u)\|_{\left(x^{*}(k), u^{*}(k)\right)}\right)
$$

for all $(x, u) \in \mathbb{X}(k) \times \mathbb{U}(k, x)$.

Note that the inequalities (10) and (12) imply that $\tilde{\ell}\left(k, x^{*}(k), u^{*}(k)\right)=0$ for each $k \in \mathbb{N}_{0}$. 
The following preliminary result shows that an optimal trajectory starting in a neighbourhood of the optimal pair $\left(x^{*}, u^{*}\right)$ will stay near the optimal pair for some time.

Lemma 1 Suppose that the system (1) is strictly dissipative and that Assumptions 1, 2 and 3 hold. Then there exist $N_{1}>0, R \geq N / 2$ and $\eta: \mathbb{N} \times \mathbb{R}_{0}^{+} \rightarrow \mathbb{R}_{0}^{+}$with $\eta(N, r) \rightarrow 0$ if $N \rightarrow \infty$ and $r \rightarrow 0$, such that for each $k>0$ the open loop optimal trajectories with horizon $N \geq N_{1}$ starting in $x_{1} \in \mathscr{B}_{\delta_{c}}\left(x^{*}(k)\right)$ satisfy

$$
\left|\left(x_{u_{N, x_{1}}^{*}}\left(j ; k, x_{1}\right), u_{N, x_{1}}^{*}(j)\right)\right|_{\left(x^{*}(k+j), u^{*}(k+j)\right)} \leq \eta\left(N,\left\|x_{1}-x^{*}(k)\right\|\right)
$$

for all $j \in\{0, \ldots, R\}$ and $\delta_{c}$ from Assumption 2 .

Proof. ${ }^{5}$ Let $k \in \mathbb{N}_{0}$. Choose an arbitrary $x_{1} \in \mathscr{B}_{\delta_{c}}\left(x^{*}(k)\right)$. By Theorem 1 we know that for the optimal trajectory $x_{u_{N, x_{1}}^{*}}\left(\cdot ; k, x_{1}\right)$ the finite horizon turnpike property holds. This means we can choose $0<\varepsilon \leq \delta_{c}$ and $N, P \leq N-2 d$ ( $d$ from Assumption 2), such that there are at least $N-P \geq 2 d$ time instants $j \in\{0, \ldots, N\}$ at which

$$
\left|\left(x_{u_{N, x_{1}}^{*}}\left(j ; k, x_{1}\right), u_{N, x_{1}}^{*}(j)\right)\right|_{\left(x^{*}(k+j), u^{*}(k+j)\right)} \leq \sigma(P) \leq \varepsilon
$$

holds. In particular we also have

$$
\left\|x_{u_{N, x_{1}}^{*}}\left(j ; k, x_{1}\right)-x^{*}(k+j)\right\| \leq \varepsilon \leq \delta_{c}
$$

for those time instants. Let $R$ denote the largest such time index and note that $R \geq$ $N-P \geq 2 d$.

We now construct a control sequence $\bar{u} \in \mathbb{U}^{N}$ as follows: By applying Assumption 2 with $x=x_{1}, y=x^{*}(k+d)$ we know that there exists a control sequence $u_{1} \in \mathbb{U}^{d}$ with $x_{u_{1}}\left(d ; k, x_{1}\right)=x^{*}(k+d)$. We define $\bar{u}(j)=u_{1}(j)$ for $j \in\{0, \ldots, d-1\}$. For $j \in$ $\{d, \ldots, R-d-1\}$ we choose $\bar{u}(j)=u^{*}(k+j)$, and thus get $x_{\bar{u}}(R-d)=x^{*}(k+R-$ $d)$. Using Assumption 2 again, this time with $x=x^{*}(k+R-d) \in \mathscr{B}_{\delta_{c}}\left(x^{*}(k+R-d)\right)$ and $y=x_{u_{N, x_{1}}^{*}}\left(R, x_{1}\right) \in \mathscr{B}_{\delta_{c}}\left(x^{*}(k+R)\right)$, we obtain the control sequence $u_{2} \in \mathbb{U}^{d}$. We finish by defining $\bar{u}(j)=u_{2}(j-R+d)$ for $j \in\{R-d, \ldots, R-1\}$ and $\bar{u}(j)=u_{N, x_{1}}^{*}(j)$ for $j \in\{R, \ldots, N-1\}$.

Observe that by construction the trajectories $x_{\bar{u}}\left(j ; k, x_{1}\right)$ and $x_{u_{N, x_{1}}^{*}}\left(j ; k, x_{1}\right)$ coincide for $j \in\{R, \ldots, N\}$. Because of the optimality principle, and because $x_{u_{N, x_{1}}^{*}}\left(j ; k, x_{1}\right)$ is the final piece of an optimal trajectory for $j \in\{R, \ldots, N\}$, the initial pieces of the control sequences $u_{N, x_{1}}^{*}$ and $\bar{u}$ up to time $R-1$ satisfy

$$
J_{R}\left(k, x_{1}, u_{N, x_{1}}^{*}\right) \leq J_{R}\left(k, x_{1}, \bar{u}\right)
$$

as well as

$$
\hat{J}_{R}\left(k, x_{1}, u_{N, x_{1}}^{*}\right) \leq \hat{J}_{R}\left(k, x_{1}, \bar{u}\right) .
$$

\footnotetext{
5 The proof uses a construction similar to the one of Lemma 6.3 in [8].
} 
Now consider the modified cost functionals $\tilde{J}_{R}$. From (11) with $N=R$ and the fact that $x_{\bar{u}}\left(R, x_{1}\right)=x_{u_{N, x_{1}}^{*}}\left(R, x_{1}\right)$ it follows that

$$
\begin{aligned}
\tilde{J}_{R}\left(k, x_{1}, u_{N, x_{1}}^{*}\right) & =\hat{J}_{R}\left(k, x_{1}, u_{N, x_{1}}^{*}\right)+\lambda\left(k, x_{1}\right)-\lambda\left(k+R, x_{u_{N, x_{1}}^{*}}\left(R ; k, x_{1}\right)\right) \\
& \stackrel{(13)}{\leq} \hat{J}_{R}\left(k, x_{1}, \bar{u}\right)+\lambda\left(k, x_{1}\right)-\lambda\left(k+R, x_{u_{N, x_{1}}^{*}}\left(R ; k, x_{1}\right)\right) \\
& =\hat{J}_{R}\left(k, x_{1}, \bar{u}\right)+\lambda\left(k, x_{1}\right)-\lambda\left(k+R, x_{\bar{u}}\left(R ; k, x_{1}\right)\right) \\
& =\tilde{J}_{R}\left(k, x_{1}, \bar{u}\right) .
\end{aligned}
$$

We abbreviate $r:=\left\|x_{1}-x^{*}(k)\right\|$. From the construction of $\bar{u}$ we know that

$$
\left\|x_{\bar{u}}\left(j ; k, x_{1}\right)-x^{*}(k+j)\right\| \leq \gamma_{x}(r) \text { and }\left\|\bar{u}(j)-u^{*}(k+j)\right\| \leq \gamma_{u}(r)
$$

for $j=\{0, \ldots, d-1\}$, and similarly $\left\|x_{\bar{u}}\left(j ; k, x_{1}\right)-x^{*}(k+j)\right\| \leq \gamma_{x}(\varepsilon)$ as well as $\left\|\bar{u}(j)-u^{*}(k+j)\right\| \leq \gamma_{u}(\varepsilon)$ for $j \in\{R-d, \ldots, R-1\}$. In addition, we have $x_{\bar{u}}\left(j ; k, x_{1}\right)=x^{*}(k+j)$ and $\bar{u}(j)=u^{*}(k+j)$ for $j \in\{d, \ldots, R-d-1\}$. Recalling that the modified stage cost satisfies $\tilde{\ell}\left(k, x^{*}(k), u^{*}(k)\right)=0$ and using Assumption 3 we thus get the following estimate for the modified cost functional with the control sequence $\bar{u}$ :

$$
\begin{aligned}
\tilde{J}_{R}\left(k, x_{1}, \bar{u}\right) & =\sum_{j=0}^{R-1} \tilde{\ell}\left(k+j, x_{\bar{u}}\left(j ; k, x_{1}\right), \bar{u}(j)\right) \\
& =\sum_{j=0}^{d-1} \underbrace{\tilde{\ell}\left(k+j, x_{\bar{u}}\left(j ; k, x_{1}\right), \bar{u}(j)\right)}_{\leq \alpha_{u}\left(\left|\left(x_{\bar{u}}\left(j ; k, x_{1}\right), \bar{u}(j)\right)\right|_{\left(x^{*}(k+j), u^{*}(k+j)\right)}\right)}+\underbrace{\sum_{j=d}^{R-d-1} \tilde{\ell}\left(k+j, x_{\bar{u}}\left(j ; k, x_{1}\right), \bar{u}(j)\right)}_{=0} \\
& +\sum_{j=R-d}^{R-1} \underbrace{\tilde{\ell}\left(k+j, \gamma_{\bar{u}}(r)\right.}_{\leq \gamma_{u}\left(\left|\left(x_{\bar{u}}\left(j ; k, x_{1}\right), \bar{u}(j)\right)\right|_{\left(x^{*}(k+j), u^{*}(k+j)\right)}\right)} \\
& \leq \sum_{j=0}^{d-1} \alpha_{u}(\underbrace{\left.\left|\left(x_{\bar{u}}\left(j ; k, x_{1}\right), \bar{u}(j)\right)\right|_{\left(x^{*}(k+j), u^{*}(k+j)\right)}\right)}_{\leq \gamma_{x}(\varepsilon)+\gamma_{u}(\varepsilon)} \\
& +\sum_{j=R-d}^{R-1} \alpha_{u}(\underbrace{\left|\left(x_{\bar{u}}\left(j ; k, x_{1}\right), \bar{u}(j)\right)\right|_{\left(x^{*}(k+j), u^{*}(k+j)\right)}}) \\
& \leq d \alpha_{u}\left(\gamma_{x}(r)+\gamma_{u}(r)\right)+d \alpha_{u}\left(\gamma_{x}(\varepsilon)+\gamma_{u}(\varepsilon)\right)
\end{aligned}
$$

Now assume that $\left|\left(x_{u_{N, x_{1}}^{*}}\left(\tilde{j} ; k, x_{1}\right), u_{N, x_{1}}^{*}(\tilde{j})\right)\right|_{\left(x^{*}(k+\tilde{j}), u^{*}(k+\tilde{j})\right)} \geq \Delta$ for some $\tilde{j} \in$ $\{0, \ldots, R-1\}$ and $\Delta>\alpha_{l}^{-1}\left(d \alpha_{u}\left(\gamma_{x}(r)+\gamma_{u}(r)\right)+d \alpha_{u}\left(\gamma_{x}(\varepsilon)+\gamma_{u}(\varepsilon)\right)\right)$. By summing up to time $R$ the modified stage cost for the control sequence $u_{N, x_{1}}^{*}$ and using (10) and (14) we get the estimate 


$$
\begin{aligned}
\tilde{J}_{R}\left(k, x_{1}, u_{N, x_{1}}^{*}\right) & =\sum_{j=0}^{R-1} \tilde{\ell}\left(k+j, x_{u_{N, x_{1}}^{*}}\left(j ; k, x_{1}\right), u_{N, x_{1}}^{*}(j)\right) \\
& \stackrel{(10)}{\geq} \sum_{j=0}^{R-1} \alpha_{l}\left(\left|\left(x_{u_{N, x_{1}}^{*}}\left(j ; k, x_{1}\right), u_{N, x_{1}}^{*}(j)\right)\right|_{\left(x^{*}(k+j), u^{*}(k+j)\right)}\right) \\
& \geq \alpha_{l}(\underbrace{\left.\left|\left(x_{u_{N, x_{1}}^{*}}\left(\tilde{j} ; k, x_{1}\right), u_{N, x_{1}}^{*}(\tilde{j})\right)\right|_{\left(x^{*}(k+\tilde{j}), u^{*}(k+\tilde{j})\right)}\right)}_{>\Delta} \\
& >d \alpha_{u}\left(\gamma_{x}(r)+\gamma_{u}(r)\right)+d \alpha_{u}\left(\gamma_{x}(\varepsilon)+\gamma_{u}(\varepsilon)\right) \\
& \stackrel{(14)}{\geq} \tilde{J}_{R}\left(k, x_{1}, \bar{u}\right) .
\end{aligned}
$$

But this contradicts (14) and thus we get $\Delta \leq \alpha_{l}^{-1}\left(d \alpha_{u}\left(\gamma_{x}(r)+\gamma_{u}(r)\right)+d \alpha_{u}\left(\gamma_{x}(\varepsilon)+\right.\right.$ $\left.\left.\gamma_{u}(\varepsilon)\right)\right)$. Finally, choose $\varepsilon=\sigma\left(\frac{N}{2}\right)$, which satisfies $\varepsilon \rightarrow 0$ for $N \rightarrow \infty$, and define $\eta(N, r):=\alpha_{l}^{-1}\left(d \alpha_{u}\left(\gamma_{x}(r)+\gamma_{u}(r)\right)+d \alpha_{u}\left(\gamma_{x}(\varepsilon)+\gamma_{u}(\varepsilon)\right)\right)$. By choice of $R$ we know that $R \geq N-P$, which for $P=\frac{N}{2}$ yields the assertion, i.e. $R \geq \frac{N}{2}$. It remains to ensure that $N-P=\frac{N}{2} \geq 2 d$ as well as $\varepsilon \leq \delta_{c}$, which can be achieved by setting $N_{1} \geq \max \left\{4 d, 2 \sigma^{-1}\left(\delta_{c}\right)\right\}$.

As a final assumption in order to prove continuity of the optimal value function we require the stage cost to be continuous.

Assumption 4 (Continuity of the stage cost) We assume that the function $\ell$ is continuous in the sense that there exists $\eta_{\ell} \in \mathscr{K}_{\infty}$ such that for each $k \in \mathbb{N}_{0}$ and each compact set $\mathbb{Y} \subseteq \mathbb{X}(k) \times \mathbb{U}(k)$ the inequality

$$
\left|\ell(k, x, u)-\ell\left(k, x^{\prime}, u^{\prime}\right)\right| \leq \eta_{\ell}\left(|(x, u)|_{\left(x^{\prime}, u^{\prime}\right)}\right)
$$

holds for all $(x, u),\left(x^{\prime}, u^{\prime}\right) \in \mathbb{Y}$.

\section{Theorem 2 (Continuity property of the optimal value function)}

If the optimal control problem (2) is strictly dissipative and Assumptions 1 - 4 are satisfied, then the optimal value function is (approximately) continuous in the sense of Definition 5.

Proof. ${ }^{6}$ Let $k \geq 0$ and pick $\delta \in\left(0, \delta_{c}\right]$ with $\delta_{c}$ from Assumption 2. To shorten the notation we write $x_{1}=x^{*}(k)$ and choose $x_{2} \in \mathscr{B}_{\delta}\left(x_{1}\right) \cap \mathbb{X}(k)$. We denote the optimal control sequence for $N$ steps starting in $x_{1}$ by $u_{N, x_{1}}^{*}$, and the one starting in $x_{2}$ by $u_{N, x_{2}}^{*}$. According to Lemma 1 we can choose $N \geq N_{1}$ sufficiently large such that both

$$
\left|\left(x_{u_{N, x_{1}}^{*}}\left(j ; k, x_{1}\right), u_{N, x_{1}}^{*}(j)\right)\right|_{\left(x^{*}(k+j), u^{*}(k+j)\right)} \leq \eta\left(N,\left\|x_{1}-x^{*}(k)\right\|\right) \leq \eta(N, \delta) \leq \delta_{c}
$$

and

$$
\left|\left(x_{u_{N, x_{2}}^{*}}\left(j ; k, x_{2}\right), u_{N, x_{2}}^{*}(j)\right)\right|_{\left(x^{*}(k+j), u^{*}(k+j)\right)} \leq \eta\left(N,\left\|x_{2}-x^{*}(k)\right\|\right) \leq \eta(N, \delta) \leq \delta_{c}
$$

\footnotetext{
${ }^{6}$ The idea is similar to the proof of Theorem 16 in [16].
} 
hold for all $j \in\{0, \ldots, R\}$. From the proof Lemma 1 we also know that $R \geq 2 d>d$.

Define $\varepsilon:=\eta(N, \delta), \hat{\delta}:=\max \{\delta, \varepsilon\}$ and let $x_{3}:=x_{u_{N, x_{1}}^{*}}\left(d ; k, x_{1}\right)$. Because of Assumption 4 we know that

$$
\begin{array}{r}
\left|\ell\left(k+j, x_{u_{N, x_{1}}^{*}}\left(j ; k, x_{1}\right), u_{N, x_{1}}^{*}(j)\right)-\ell\left(k+j, x^{*}(k+j), u^{*}(k+j)\right)\right| \\
\leq \eta_{\ell}\left(\left|\left(x_{u_{N, x_{1}}^{*}}\left(j ; k, x_{1}\right), u_{N, x_{1}}^{*}(j)\right)\right|_{\left(x^{*}(k+j), u^{*}(k+j)\right)}\right) \leq \eta_{\ell}(\varepsilon) .
\end{array}
$$

This leads to the estimate

$$
\sum_{j=0}^{d-1} \underbrace{\ell\left(k+j, x_{u_{N, x_{1}}^{*}}\left(j ; k, x_{1}\right), u_{N, x_{1}}^{*}(j)\right)}_{\geq \ell\left(k+j, x^{*}(k+j), u^{*}(k+j)\right)-\eta_{\ell}(\varepsilon)} \geq J_{d}^{*}(k)-d \eta_{\ell}(\varepsilon) .
$$

Furthermore, we can apply Assumption 2 with $x=x_{2}, y=x_{3}$ to conclude that there exists a control sequence $u_{1} \in \mathbb{U}^{d}$ such that $x_{u_{1}}\left(d, x_{2}\right)=x_{3}$ and the estimate

$$
\begin{aligned}
& \left|\ell\left(k+j, x_{u_{1}}\left(j, x_{2}\right), u_{1}(j)\right)-\ell\left(k+j, x^{*}(k+j), u^{*}(k+j)\right)\right| \\
& \quad \leq \gamma_{c}\left(\max \left\{\left\|x_{2}-x^{*}(k)\right\|,\left\|x_{3}-x^{*}(k+d)\right\|\right\}\right) \leq \gamma_{c}(\hat{\delta})
\end{aligned}
$$

holds for all $j \in\{0, \ldots, d-1\}$. This yields

$$
\sum_{j=0}^{d-1} \underbrace{\ell\left(k+j, x_{u_{1}}\left(j ; k, x_{2}\right), u_{1}(j)\right)}_{\leq \ell\left(k+j, x^{*}(k+j), u^{*}(k+j)\right)+\gamma_{c}(\hat{\boldsymbol{\delta}})} \leq J_{d}^{*}(k)+d \gamma_{c}(\hat{\delta}) .
$$

Now define a control sequence $\bar{u} \in \mathbb{U}^{N}$ by $\bar{u}(j)=u_{1}(j)$ for $j \in\{0, \ldots, d-1\}$ and $\bar{u}(j)=u_{N, x_{1}}^{*}(j)$ for $j \in\{d, \ldots, N-1\}$ and note that by construction of $\bar{u}$ the trajectories of $x_{\bar{u}}\left(j ; k, x_{2}\right)$ and $x_{u_{N, x_{1}}^{*}}\left(j ; k, x_{1}\right)$ coincide for $j \in\{d, \ldots, N\}$. Thus we get

$$
\begin{aligned}
V_{N}\left(k, x_{2}\right) \leq & J_{N}\left(k, x_{1}, \bar{u}\right) \\
= & \sum_{j=0}^{d-1} \ell\left(k+j, x_{\bar{u}}\left(j ; k, x_{2}\right), \bar{u}(j)\right)+\sum_{j=d}^{N-1} \ell\left(k+j, x_{\bar{u}}\left(j ; k, x_{2}\right), \bar{u}(j)\right) \\
= & \underbrace{\sum_{j=0}^{d-1} \ell\left(k+j, x_{u_{1}}\left(j ; k, x_{2}\right), u_{1}(j)\right)}_{\leq J_{d}^{*}(k)+d \gamma_{c}(\hat{\boldsymbol{\delta}})}-\underbrace{\sum_{j=0}^{d-1} \ell\left(k+j, x_{u_{N, x_{1}}^{*}}\left(j ; k, x_{1}\right), u_{N, x_{1}}^{*}(j)\right)}_{\geq J_{d}^{*}(k)-d \eta_{\ell}(\varepsilon)} \\
& +\sum_{j=0}^{N-1} \ell\left(k+j, x_{u_{N, x_{1}}^{*}}\left(j ; k, x_{1}\right), u_{N, x_{1}}^{*}(j)\right) \\
\leq & V_{N}\left(k, x_{1}\right)+d\left(\gamma_{c}(\hat{\boldsymbol{\delta}})+\eta_{\ell}(\varepsilon)\right) .
\end{aligned}
$$

Setting $\left.\tilde{\gamma}_{V}(N, \delta)=d\left(\gamma_{c}(\hat{\boldsymbol{\delta}})+\eta_{\ell}(\varepsilon)\right)\right)$ and using the definition of $\hat{V}_{N}$ then yields 


$$
\hat{V}_{N}\left(k, x_{2}\right) \leq \hat{V}_{N}\left(k, x_{1}\right)+\tilde{\gamma}_{V}(N, \delta) .
$$

Observe that $\tilde{\gamma}_{V} \rightarrow 0$ if both $N \rightarrow \infty$ and $\delta \rightarrow 0$. Finally, to get the required monotonicity we define

$$
\gamma_{V}(N, r):=\sup _{\tilde{N} \geq N, \tilde{\boldsymbol{\delta}} \leq r} \tilde{\gamma}_{V}(\tilde{N}, \tilde{\boldsymbol{\delta}}),
$$

for which (16) remains true.

The converse inequality follows by exchanging the roles of $x_{1}$ and $x_{2}$ which concludes the proof.

\section{Optimality Conditions imply Dissipativity}

In this section we show how strict dissipativity can be established if optimality conditions for the infinite horizon optimal control problem (2) are satisfied. The proof extends those for discounted and non-discounted time-invariant optimal control problems, see [9] and [5]. The optimality conditions in the literature which most easily lead to the desired result are those derived in [4, Theorem 2.2], which we will hence use in the sequel. However, we believe that using other optimality conditions strict dissipativity can be proved, too. We will elaborate more on this with respect to the results stated in [2] at the end of the section.

To be consistent with [4, Theorem 2.2], let us assume that $X=\mathbb{R}^{n}$ and $U=\mathbb{R}^{m}$ and that no constraints are imposed on the state and control variables. We first define the Hamiltonian which is the key ingredient for deriving optimality conditions.

Definition 8 (Hamiltonian) For all times $k \in \mathbb{N}_{0}$ the Hamiltonian $H_{k}: X \times U \times$ $\mathbb{R}^{n} \times \mathbb{R} \rightarrow \mathbb{R}$ of problem (2) is defined by

$$
H_{k}(x, u, p, \eta):=-\eta \ell(k, x, u)+p^{T} f(k, x, u) .
$$

For the readers' convenience we state [4, Theorem 2.2] in our notation. Note that the sign of $\ell$ has been changed in the definition above and theorem below because we are considering minimization problems, here.

Theorem 3 Let $\left(x^{*}, u^{*}\right)$ be an overtaking optimal pair for (2). If it holds:

1. For all $k \in \mathbb{N}_{0}$ the functions $\ell(k, \cdot, \cdot)$ and $f(k, \cdot, \cdot)$ are continuous on a neighborhood of $\left(x^{*}, u^{*}\right)$ and differentiable at $\left(x^{*}, u^{*}\right)$.

2. For all $k \in \mathbb{N}_{0}$ the partial differential $\frac{\partial f}{\partial x}\left(k, x^{*}(k), u^{*}(k)\right)$ is invertible.

Then, there are $\eta_{0} \in \mathbb{R}$, and $p_{k+1} \in \mathbb{R}^{n}$ for all $k \in \mathbb{N}_{0}$ satisfying the following conditions:

(i) $\left(\eta_{0}, p_{1}\right) \neq(0,0)$.

(ii) $\eta_{0} \geq 0$.

(iii) For all $k \in \mathbb{N}_{0}$ it holds 


$$
p_{k}=p_{k+1}^{T} \frac{\partial f}{\partial x}\left(k, x^{*}(k), u^{*}(k)\right)-\eta_{0} \frac{\partial \ell}{\partial x}\left(k, x^{*}(k), u^{*}(k)\right) .
$$

(iv) For all $k \in \mathbb{N}_{0}$ it holds $\frac{\partial H_{k}}{\partial u}\left(x^{*}(k), u^{*}(k), p_{k+1}, \eta_{0}\right)=0$.

In what follows, structural assumptions on the optimal control problems are imposed.

Assumption 5 We assume that the dynamics $f(k, \cdot, \cdot)$ are affine for each $k \in \mathbb{N}_{0}$. We also assume that there is $\kappa \in \mathbb{R}_{>0}$ and $F \in \mathscr{K}_{\infty}$ such that for all $k \in \mathbb{N}_{0}$ it holds

$$
\begin{aligned}
\ell\left(k, t\left(x_{1}, u_{1}\right)+(1-t)\left(x_{2}, u_{2}\right)\right) \leq & t \ell\left(k, x_{1}, u_{1}\right)+(1-t) \ell\left(k, x_{2}, u_{2}\right) \\
& -\frac{\kappa}{2} t(1-t) F\left(\left\|\left(x_{1}, u_{1}\right)-\left(x_{2}, u_{2}\right)\right\|\right)
\end{aligned}
$$

for all $\left(x_{1}, u_{1}\right),\left(x_{2}, u_{2}\right) \in X \times U$ and $t \in[0,1]$.

Remark 11 . We call the property introduced in Assumption 5 uniform strict convexity of $\ell$ wrt $\kappa$ and $F$. The word uniform refers to the fact that $\kappa$ and $F$ do not depend on the time $k$.

2. It follows from the definitions, that strong convexity (see e.g. [17] for a definition) implies (17) and this property itself implies strict convexity.

Theorem 4 (Optimality conditions imply strict dissipativity) Let Assumption 5 and those of Theorem 3 hold. If $\eta_{0} \neq 0$ and $\sup _{k \in \mathbb{N}_{0}}\left\|p_{k}\right\|<\infty$, then the optimal control problem (2) is strictly dissipative on every bounded set $\mathbb{X}_{0}$ wrt supply rate $s(k, x, u)=\hat{\ell}(k, x, u)$ and optimal pair $\left(x^{*}, u^{*}\right)$.

Proof. In order to prove strict dissipativity we have to verify that there is $\alpha \in \mathscr{K}_{\infty}$ and a storage function $\lambda$ such that (6) holds. We claim that making the ansatz $\lambda(k, x)=\frac{1}{\eta_{0}} p_{k}^{T}\left(x-x^{*}(k)\right)$ yields the desired property. Note that the restriction to bounded sets $\mathbb{X}_{0}$ is needed here in order to ensure that $\lambda$ is bounded from below as required in Definition 6.

Let $\mathbb{X}_{0}$ be an arbitrary bounded set in $\mathbb{R}^{n}$. This yields boundedness of $\lambda$. Conditions (iii) and (iv) in Theorem 3 read

(iii) $\forall k \in \mathbb{N}_{0}: p_{k}=-\eta_{0} \frac{\partial \ell}{\partial x}\left(k, x^{*}(k), u^{*}(k)\right)+p_{k+1}^{T} \frac{\partial f}{\partial x}\left(k, x^{*}(k), u^{*}(k)\right)$ and

(iva) $\forall k \in \mathbb{N}_{0}:-\eta_{0} \frac{\partial \ell}{\partial u}\left(k, x^{*}(k), u^{*}(k)\right)+p_{k+1}^{T} \frac{\partial f}{\partial u}\left(k, x^{*}(k), u^{*}(k)\right)=0$.

Let us consider the modified stage cost $\tilde{\ell}$ (cf. Definition 7) using our ansatz for the storage function:

$$
\begin{aligned}
\tilde{\ell}(k, x, u)=\hat{\ell}(k, x, u) & +\frac{1}{\eta_{0}} p_{k}^{T}\left(x-x^{*}(k)\right)-\frac{1}{\eta_{0}} p_{k+1}^{T}\left(f(k, x, u)-x^{*}(k+1)\right) \\
=\ell(k, x, u) & -\ell\left(k, x^{*}(k), u^{*}(k)\right) \\
+ & \frac{1}{\eta_{0}} p_{k}^{T}\left(x-x^{*}(k)\right)-\frac{1}{\eta_{0}} p_{k+1}^{T}\left(f(k, x, u)-x^{*}(k+1)\right)
\end{aligned}
$$

\footnotetext{
${ }^{7}$ This means that dissipativity holds for all $x \in \mathbb{X}_{0}$.
} 
Since $\ell$ is uniformly strictly convex wrt $\kappa$ and $F, p_{k}$ linear and $f$ affine for each $k$, the modified cost $\tilde{\ell}$ is uniformly strictly convex wrt $\kappa$ and $F$ (and in particular strictly convex for all $\left.k \in \mathbb{N}_{0}\right)$. This means that a point $(\bar{x}(k), \bar{u}(k))$ satisfying $\frac{\partial \tilde{\ell}}{\partial x}(k, \bar{x}(k), \bar{u}(k))=\frac{\partial \tilde{\ell}}{\partial u}(k, \bar{x}(k), \bar{u}(k))=0$ is a unique strict minimizer of $\tilde{\ell}(k, \cdot, \cdot)$. Let us therefore consider the partial derivatives of $\tilde{\ell}$. For all $k \in \mathbb{N}_{0}$ we have

$$
\begin{aligned}
& \frac{\partial \tilde{\ell}}{\partial x}(k, x, u)=\frac{\partial \ell}{\partial x}(k, x, u)+\frac{1}{\eta_{0}} p_{k}-\frac{1}{\eta_{0}} p_{k+1}^{T} \frac{\partial f}{\partial x}(k, x, u) \text { and } \\
& \frac{\partial \tilde{\ell}}{\partial u}(k, x, u)=\frac{\partial \ell}{\partial u}(k, x, u)-\frac{1}{\eta_{0}} p_{k+1}^{T} \frac{\partial f}{\partial u}(k, x, u) .
\end{aligned}
$$

Now plugging in $\left(x^{*}(k), u^{*}(k)\right)$ and conditions (iii) and (iva) for the first and second equation, respectively, we obtain

$$
\frac{\partial \tilde{\ell}}{\partial x}\left(k, x^{*}(k), u^{*}(k)\right)=0 \text { and } \frac{\partial \tilde{\ell}}{\partial u}\left(k, x^{*}(k), u^{*}(k)\right)=0 .
$$

For each $k \in \mathbb{N}_{0}$ the point $\left(x^{*}(k), u^{*}(k)\right)$ is thus the unique strict minimizer of $\tilde{\ell}$ at time $k$. By definition of the modified stage cost $\tilde{\ell}$ we have

$$
\begin{aligned}
\tilde{\ell}\left(k, x^{*}(k), u^{*}(k)\right) & =\hat{\ell}\left(k, x^{*}(k), u^{*}(k)\right)+\lambda\left(k, x^{*}(k)\right)-\lambda\left(k+1, f\left(k, x^{*}(k), u^{*}(k)\right)\right) \\
& =p_{k}^{T}\left(x^{*}(k)-x^{*}(k)\right)-p_{k+1}^{T}\left(f\left(k, x^{*}(k), u^{*}(k)\right)-x^{*}(k+1)\right) \\
& =0 .
\end{aligned}
$$

Fix an arbitrary $t \in(0,1)$. For $k \in \mathbb{N}_{0}$ consider an arbitrary point $(x, u) \in X \times U$. We define $(\bar{x}, \bar{u}):=t(x, u)+(1-t)\left(x^{*}(k), u^{*}(k)\right) \in X \times U$. Assumption 5 implies

$$
\begin{aligned}
\tilde{\ell}(k, \bar{x}, \bar{u})+ & \frac{\kappa}{2} t(1-t) F\left(\left\|(x, u)-\left(x^{*}(k), u^{*}(k)\right)\right\|\right) \\
& \leq t \tilde{\ell}(k, x, u)+(1-t) \tilde{\ell}\left(k, x^{*}(k), u^{*}(k)\right)=t \tilde{\ell}(k, x, u) \\
\Rightarrow \tilde{\ell}(k, x, u) & >\frac{1}{t} \tilde{\ell}\left(k, x^{*}(k), u^{*}(k)\right)+\frac{\kappa}{2}(1-t) F\left(\left\|(x, u)-\left(x^{*}(k), u^{*}(k)\right)\right\|\right) \\
& =\frac{\kappa}{2}(1-t) F\left(\left\|(x, u)-\left(x^{*}(k), u^{*}(k)\right)\right\|\right) .
\end{aligned}
$$

This implies (6) if we set $\alpha(r):=\frac{\kappa}{2}(1-t) F(r)$, which is of class $\mathscr{K}_{\infty}$ because $F \in \mathscr{K}_{\infty}$ and $\frac{\kappa}{2}(1-t) \in \mathbb{R}_{>0}$.

Remark 2 The assumption of $\ell$ being uniformly strictly convex is needed in order to establish that $\alpha \in \mathscr{K}_{\infty}$ in (6) does not depend on the time $k$.

As indicated at the beginning of the section the optimality conditions of the reference [4, Theorem 2.2] fit our purpose very well but are just exemplary and we conjecture that alternative conditions can also be taken to establish strict dissipativity and thus the turnpike property. We will point out similarities and differences of the conditions above with those in [2]. Firstly, let us mention that an important 
part of [2] is that the authors are able to establish a transversality condition. Such conditions are a valuable tool to restrict the set of candidates of optimal solutions to the infinite-horizon optimal control problem and, moreover, can be used in order to ensure $\sup _{k \in \mathbb{N}_{0}}\left\|p_{k}\right\|<\infty$ in Theorem 4 . A comparable result does not exist in [4, Section 2.2] (but in other results in that reference).

The assumptions that are imposed in $[2,4]$ are in general difficult to compare. However, the main assumption (Assumption A) in [2] can be simplified if Condition 2 in Theorem 3 holds. Moreover, reference [2] assumes weakly overtaking optimality whereas the theorem we used from [4] assumes overtaking optimality. The statements in the theorems are strongly related: Condition (iii) in Theorem 3 is the same as [2, Corollary 2.3], and Condition (iv) is similar to the maximum condition in [2, Theorem 2.2], that reads (adapted to our notation)

$$
\forall k \in \mathbb{N}_{0}:\left(-\frac{\partial \ell}{\partial u}\left(k, x^{*}(k), u^{*}(k)\right)+p_{k+1}^{T} \frac{\partial f}{\partial u}\left(k, x^{*}(k), u^{*}(k)\right)\right) v \leq 0
$$

$\forall v \in T_{U_{k}}\left(u^{*}(k)\right)$. The set $T_{U_{k}}\left(u^{*}(k)\right)$ denotes the Bouligand tangent cone of $U_{k}$ (the constraint set for $u$ at time $k$ in [2]) at point $u^{*}(k)$. Certainly, (18) is obtained under weaker assumptions than [4, Theorem 2.2], yet it also yields a weaker statement and it is currently an open question whether it is still sufficient to prove strict dissipativity.

\section{Example}

In this section we provide an example of a time-varying optimal control problem, that was introduced in [12]. It can be interpreted as a very simple room heating/cooling model that has to react to external influences (the weather). We will verify that the example meets the assumptions needed for strict dissipativity and for the turnpike property. The latter will also be illustrated by means of numerical simulations.

The system dynamics is given by

$$
f: \mathbb{N}_{0} \times \mathbb{R} \times \mathbb{R} \rightarrow \mathbb{R}, f(k, x, u)=x+u+w_{k},
$$

with $w_{k}=-2 \sin \left(\frac{k \pi}{12}\right)+a_{k}$ and in which the $a_{k}$ are iid random numbers on the interval $\left[-\frac{1}{4}, \frac{1}{4}\right]$. In a physical interpretation of the example the state $x$ corresponds to the temperature within a room, the control $u$ to the heating/cooling and the time-varying data $w_{k}$ to the changes of the external temperature over time that also influence the inside temperature. The stage cost of the system is

$$
\ell(k, x, u)=u^{2}+\varepsilon x^{2},
$$

for $0<\varepsilon \ll 1$. Note that the term $\varepsilon x^{2}$ is a regularization term that renders the original cost $u^{2}$, that was used in [12], strictly convex wrt $x$ and $u$. However, nu- 
merical experiments show, that the optimal trajectories for both versions of $\ell$ do not differ for sufficiently small $\varepsilon$. The system has to be operated subject to the control constraints $\mathbb{U}(k)=[-3,3]$ and the state constraints $\mathbb{X}(k)=[-1 / 2,1 / 2]$ if $k \in\left[24 j+12,24(j+1), j \in \mathbb{N}_{0}\right.$ and $\mathbb{X}(k)=[-2,2]$ if $k \in[24 j, 24 j+12)$. We assume that we have a perfect prediction of the external influence $w_{k}$, which means that its values are known whenever we optimize. Since a correct weather forecast is hardly possible for a few days, let alone on an infinite horizon, this may not be realistic. However, a verification of the turnpike property would allow us to apply model predictive control, in which only finite horizon problems of moderate horizon length have to be solved.

In what follows, we aim to verify the assumptions of Theorem 4. Since this result was stated for unconstrained problems, we first rewrite the example above using penalty functions $b_{1}: \mathbb{N}_{0} \times \mathbb{R} \rightarrow \mathbb{R}_{\geq 0}$ and $b_{2}: \mathbb{N}_{0} \times \mathbb{R} \rightarrow \mathbb{R}_{\geq 0}$. Then, the reformulated stage cost is given as follows (the dynamics remain unchanged):

$$
\begin{aligned}
L(k, x, u) & :=l(k, x, u)+b_{1}(k, x)+b_{2}(k, u), \\
b_{1}(k, x) & =\left\{\begin{array}{ll}
c_{x}(|x|-2)^{4} & , x \notin[-2,2] \\
0 & , x \in[-2,2]
\end{array}, k \in[24 j, 24 j+12), j \in \mathbb{N}_{0},\right. \\
b_{1}(k, x) & =\left\{\begin{array}{ll}
c_{x}(|x|-1 / 2)^{4} & , x \notin[-1 / 2,1 / 2] \\
0 & , x \in[-1 / 2,1 / 2]
\end{array}, k \in[24 j+12,24(j+1)), j \in \mathbb{N}_{0},\right. \\
b_{2}(k, u) & =\left\{\begin{array}{ll}
c_{u}(|u|-3)^{4} & , u \notin[-3,3] \\
0 & , u \in[-3,3]
\end{array}, k \in \mathbb{N}_{0},\right.
\end{aligned}
$$

with $c_{x}$ and $c_{u} \in \mathbb{R}_{>0}$.

We claim, that the reformulated optimal control problem satisfies Assumption 5. It is clear that for predictible $a_{k}$ the dynamics are affine for each $k \in \mathbb{N}_{0}$. The Hessian of the stage cost reads

$$
H_{(x, u)} L(k, x, u)=\left(\begin{array}{cc}
2 \varepsilon+\frac{d^{2} b_{1}}{d x^{2}}(k, x) & 0 \\
0 & 2+\frac{d^{2} b_{2}}{d u^{2}}(k, u)
\end{array}\right) .
$$

It is easily seen, that $\frac{d^{2} b_{1}}{d x^{2}}(k, x) \geq 0$ and $\frac{d^{2} b_{2}}{d u^{2}}(k, u) \geq 0$ for all $k \in \mathbb{N}_{0}, x \in \mathbb{R}$ and $u \in \mathbb{R}$ such that we can conclude positive semidefiniteness of the matrix $H_{(x, u)} L(k, x, u)-$ $2 \varepsilon I$, in which $I$ is the identity matrix of dimension 2 . For two times continuously differentiable functions this property is equivalent to $L$ being strongly convex wrt $2 \varepsilon$ (see e.g. [17]) for all $k \in \mathbb{N}_{0}$ and this implies uniform strict convexity of $L$ wrt $\kappa=2 \varepsilon$ and $F(r)=r^{2}$.

Let us now check the assumptions of Theorem 3. Clearly, the continuity and differentiability requirements are met. The second condition also holds because $\frac{\partial f}{\partial x}(k, x, u)=1$. For this example it moreover holds, that $\eta_{0} \neq 0$ : If $\eta_{0}=0$ then Theorem 3 yields that $p_{1} \neq 0$. From condition (iii) applied to this example we get $p_{k}=p_{k+1}$ for all $k \in \mathbb{N}_{0}$. This contradicts (iva), which in case $\eta_{0}=0$ implies 
$p_{k+1}=0$. It is left to show that the adjoints $p_{k}$ are bounded. A formal proof appears technically involved, however, we can give evidence why it is reasonable to expect bounded $p_{k}$. The adjoint $p_{k}$ is a measurement of how much the value of the trajectory differs from the optimal value if the trajectory value at time $k$ differs (slightly) from $x^{*}(k)$. In our example the absence of constraints allows to steer the trajectory to $x^{*}(k+1)$ in one step after having been disturbed at time $k$. Thus, the value of the disturbed trajectory and the optimal trajectory only differ in the first term and this difference can be estimated on bounded sets by a bound which is independent of $k$. This implies boundedness of the $p_{k}$ and thus by Theorem 3 strict dissipativity for our example.

In what follows we will investigate Assumption 1 to conclude by Theorem 1 that the example exhibits the turnpike property on any compact set $\mathbb{X}_{0} \subset \mathbb{R}^{n}$.

For the cheap reachability in Assumption 1 one first shows that the optimal pair $\left(x^{*}, u^{*}\right)$ satisfies the (uniform) estimates

$$
\left|x^{*}(k)\right| \leq \sqrt[4]{\frac{81-4 \varepsilon}{16 c_{x}}}+2
$$

and

$$
\left|u^{*}(k)\right| \leq \sqrt[4]{\frac{81-4 \varepsilon}{16 c_{u}}}+3 .
$$

The idea of the proof is as follows: We compare the cost of an admissible trajectory that is constructed such that it is constantly zero after the first time step, to the cost of the optimal pair. If the estimates above are violated this contradicts the fact that $\left(x^{*}, u^{*}\right)$ is overtaking optimal. For cheap reachability we need to show that there exists $E \in \mathbb{R}$ such that for all $k \in \mathbb{N}_{0}, x \in \mathbb{X}_{0}$ and $N \in \mathbb{N} \cup\{\infty\}$ it holds $\hat{V}_{N}(k, x) \leq E$. To see this we consider a control sequence $\tilde{u}(\cdot)$ of length $N$ given by $\tilde{u}(0)=-x+$ $x^{*}(k+1)-w_{k}, \tilde{u}(j)=u_{N-1, x^{*}(k+1)}^{\star}(j-1), j \in\{1, \ldots, N-1\}$. This yields

$$
\begin{aligned}
\hat{V}_{N}(k, x) & \leq \hat{\ell}(k, x, \tilde{u}(0))+\underbrace{\hat{V}_{N-1}\left(k+1, x^{*}(k+1)\right)}_{\leq 0} \\
& \leq \ell(k, x, \tilde{u}(0))-\underbrace{\ell\left(k, x^{*}(k), u^{*}(k)\right)}_{\geq 0} \\
& \leq \varepsilon x^{2}+\left(-x+x^{*}(k+1)-w_{k}\right)^{2}+b_{1}(k, x)+b_{2}\left(k,-x+x^{*}(k+1)-w_{k}\right) .
\end{aligned}
$$

Using compactness of $\mathbb{X}_{0}$, boundedness of $\left(w_{k}\right)_{k \in \mathbb{N}_{0}},\left(x^{*}(k)\right)_{k \in \mathbb{N}_{0}}$ and $\left(u^{*}(k)\right)_{k \in \mathbb{N}_{0}}$, the fact that the $b_{i}$ can be bounded uniformly in $k$ using (19), (20) we obtain a bound $E$ that does not depend on $k, x$ and $N$ and conclude the assertion.

We performed several numerical simulations that illustrate that the system in the example has the turnpike property. For the purpose of the simulations the trajectory of optimal operation on an infinite horizon has been approximated by computing an optimal trajectory on a large finite horizon of $N=100$ and leaving the initial value free. In the figures this trajectory is depicted in black. The regularization factor was chosen as $\varepsilon=10^{-10}$ and the penalty parameters as $c_{x}=c_{u}=10^{10}$. 


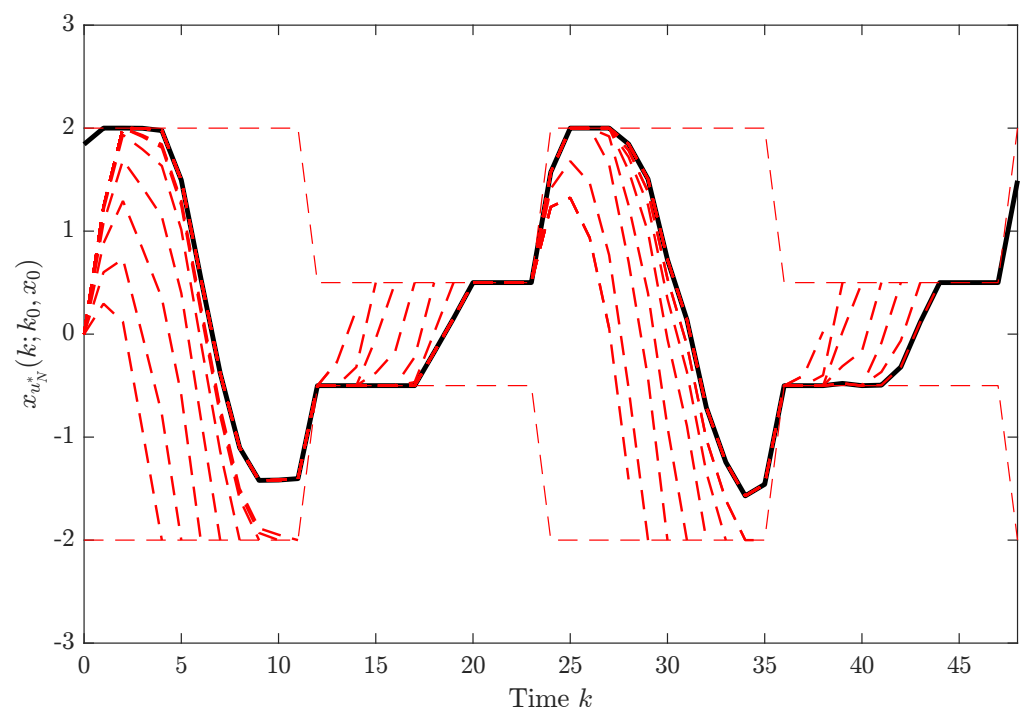

Fig. 3 Numerical simulations of the trajectory of optimal operation (black line) and open-loop trajectories of the state (dashed red lines) with different fixed initial value $x_{0}=0$ and different horizon lengths of $N$.

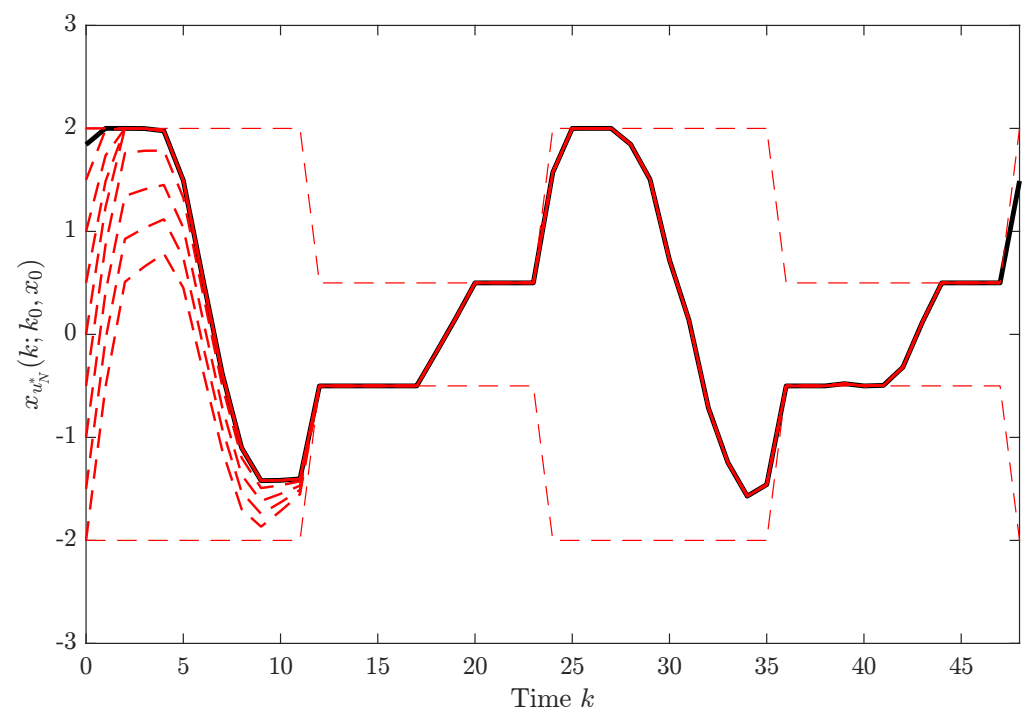

Fig. 4 Numerical simulations of the trajectory of optimal operation (black line) and open-loop trajectories of the state (dashed red lines) with different initial values $x_{0}$ and fixed horizon length of $N=48$. 
Figure 3 depicts open-loop trajectories of the state for different horizon lengths. As one can see the trajectories are close to the trajectory of optimal operation most of the time. It is also visible that the finite horizon trajectories will at some point turn away from the optimal trajectory and hit the constraints. This is due to the fact that it is cheaper to deviate from the infinite horizon optimal trajectory than it would be to stay close to it. Such a behaviour is typically observed under the turnpike property.

In Figure 4 open-loop trajectories for different initial values and fixed horizon length of $N=48$ are shown. One observes that the open-loop solutions quickly converge to the trajectory of optimal operation.

\section{References}

1. Brian D. O. Anderson and Petar V. Kokotović. Optimal control problems over large time intervals. Automatica, 23(3):355-363, 1987.

2. Sergey M. Aseev, Mikhail I. Krastanov, and Vladimir M. Veliov. Optimality conditions for discrete-time optimal control on infinite horizon. Research Report 2016-09, 2016. available online.

3. Truman F. Bewley. General equilibrium, overlapping generations models, and optimal growth theory. Harvard University Press, 2009.

4. Joël Blot and Naïla Hayek. Infinite-horizon optimal control in the discrete-time framework. Springer, 2014

5. Tobias Damm, Lars Grüne, Marleen Stieler, and Karl Worthmann. An exponential turnpike theorem for dissipative discrete time optimal control problems. SIAM Journal on Control and Optimization, 52(3):1935-1957, 2014.

6. Robert Dorfman, Paul A. Samuelson, and Robert M. Solow. Linear Programming and Economic Analysis. Dover Publications, New York, 1987. Reprint of the 1958 original.

7. David Gale. On optimal development in a multi-sector economy. Rev. Econ. Studies, 34(1):1$18,1967$.

8. Lars Grüne. Economic receding horizon control without terminal constraints. Automatica, 49(3):725-734, 2013.

9. Lars Grüne, Christopher M. Kellett, and Steven R. Weller. On a discounted notion of strict dissipativity. IFAC-PapersOnLine, 49(18):247-252, August 2016.

10. Lars Grüne, Christopher M. Kellett, and Steven R. Weller. On the relation between turnpike properties for finite and infinite horizon optimal control problems. J. Optim. Theory Appl., 2017. To appear. Online version available via doi:10.1007/s10957-017-1103-6.

11. Lars Grüne and Matthias A. Müller. On the relation between strict dissipativity and the turnpike property. Syst. Contr. Lett., 90:45-53, 2016.

12. Lars Grüne and Simon Pirkelmann. Closed-loop performance analysis for economic model predictive control of time-varying systems. Preprint, Universität Bayreuth, March 2017. Submitted.

13. Lars Grüne and Marleen Stieler. Asymptotic stability and transient optimality of economic mpc without terminal conditions. J. Proc. Control, 24(8):1187-1196, 2014.

14. Christopher M. Kellett. A compendium of comparison function results. Math Control, Sign. Syst., 26(3):339-374, 2014.

15. Lionel W. McKenzie. Optimal economic growth, turnpike theorems and comparative dynamics. In Handbook of Mathematical Economics, Vol. III, pages 1281-1355. North-Holland, Amsterdam, 1986.

16. Matthias A. Müller and Lars Grüne. Economic model predictive control without terminal constraints for optimal periodic behavior. Automatica, 70:128-139, 2016. 
17. Yurri Nesterov. Introductory Lectures on Convex Optimization: A Basic Course, volume 87 of Applied Optimization. Springer US, 1 edition, 2004.

18. Alessandro Porretta and Enrique Zuazua. Long time versus steady state optimal control. SIAM J. Control Optim., 51(6):4242-4273, 2013.

19. Emmanuel Trélat and Enrique Zuazua. The turnpike property in finite-dimensional nonlinear optimal control. J. Differential Equations, 258(1):81-114, 2015.

20. Jan C. Willems. Dissipative dynamical systems part i: General theory. Archive for Rational Mechanics and Analysis, 45(5):321-351, 1972. 